\title{
Strategies for stabilizing nitrogenous compounds in ECLSS wastewater: Top-down system design and unit operation selection with focus on bio-regenerative processes for short and long term scenarios.
}

\author{
Griffin M. Lunn ${ }^{1}$ \\ QinetiQ North America, Kennedy Space Center, FL, 32899
}

Water recycling and eventual nutrient recovery is crucial for surviving in or past low earth orbit. New approaches and system architecture considerations need to be addressed to meet current and future system requirements. This paper proposes a flexible system architecture that breaks down pretreatment steps into discrete areas where multiple unit operations can be considered. An overview focusing on the urea and ammonia conversion steps allows an analysis on each process's strengths and weaknesses and synergy with upstream and downstream processing. Process technologies to be covered include chemical pretreatment,

biological urea hydrolysis, chemical urea hydrolysis, combined nitrification-denitrification, nitrate nitrification, anammox denitrification, and regenerative ammonia absorption through struvite formation. Biological processes are considered mainly for their ability to both maximize water recovery and to produce nutrients for future plant systems. Unit operations can be considered for traditional equivalent system mass

requirements in the near term or what they can provide downstream in the form of usable chemicals or nutrients for the long term closed-loop ecological control and life support system. Optimally this would allow a system to meet the former but to support the latter without major modification.

\section{Nomenclature}

$\begin{array}{lll}\text { ISS } & = & \text { International Space Station } \\ \text { HFMB } & = & \text { Hollow Fiber Membrane Bioreactor } \\ \text { ECLSS } & = & \text { Environmental Control and Life Support System } \\ \Theta & = & \text { Residence time or Retention time } \\ \text { VOCs } & = & \text { Volatile organic compounds } \\ \text { TOC } & = & \text { Total organic Carbon } \\ \text { TN } & = & \text { Total Nitrogen } \\ \text { BOD } & = & \text { Biological Oxygen Demand } \\ \text { TDS } & = & \text { Total Dissolved Solids } \\ \text { VCD } & = & \text { Vapor Compression Distillation } \\ \text { CSTR } & = & \text { Constantly stirred tank reactor } \\ \text { PFD } & = & \text { Process Flow diagram } \\ \text { MAP } & = & \text { Magnesium Ammonium Phosphate hexahydrate } \\ \text { MP } & = & \text { Magnesium Phosphate dibasic trihydrate } \\ \text { STP } & = & \text { Standard Temperature and Pressure (1 ATM, 25C) } \\ \text { LEO } & = & \text { Low Earth Orbit } \\ \text { Caustic } & = & \text { High alkalinity solution (NaOH, K2CO3, KOH, and similar) } \\ \text { TRL } & = & \text { Technology Readiness level }\end{array}$

${ }^{1}$ Chemical Engineer Sustainable Systems Research, Mail Code ESC-53, Kennedy Space Center, FL 32899. 


\section{Introduction}

Water consists of the majority of the daily mass requirements for crewed space missions. Used for drinking, food prep, or hygiene; water is one of the most critical life support elements. Reliable water recovery is paramount for long term space exploration either on the ISS or beyond our gravity well. Advances in water recovery have allowed longer mission durations and decreased launch costs. In order to visit or even habituate extraterrestrial bodies (Mars, Europa) further advances in water recovery are needed. In this paper we will look at current state of the art water recovery systems for space applications along with new system architecture for future missions. We will also look at unit operations that fit into this new architecture and example set-ups for near and long term missions and how they integrate with plant and brine recovery systems for potential "closed loop" operation.

Wastewater in spacecraft is made up of multiple fractions. Humidity condensate is procured from scrubbers that remove excess humidity from the cabin and reclaim water contaminated with VOCs. Hygiene water stems from hand washing, food waste, and other personal care like shaving or brushing teeth. Urine is made up of obviously urine and its corresponding flush water used in spacecraft toilet systems. Future waste water stream include laundry which has been considered for the ISS. These waste streams contain high amounts of TDS,TOC, TN, and BOD; which are much higher than terrestrial waste water streams.

Early spacecraft water recovery systems focused on humidity condensate. On Salut-4 water recovery was first attempted on humidity condensate for use in food and drink preparation. This system was upgraded for MIR to handle humidity condensate and urine in separate systems. Humidity condensate was treated using absorption and catalytic oxidation while urine was simply distilled with effluents from both processes send to polishing unit operations. Lessons learned from these systems lead to the current design aboard the ISS.

\section{System Architectures}

\section{A. Current Pretreatment system}

In order for the ISS to maintain a crew of 6, water recovery was needed. Like previous Russian systems humidity condensate and urine were recycled; however hygiene water was also processed. Urine collected from toilets is pretreated using oxone/sulfuric acid or chromic acid (depending on side of the ISS) in order to reduce volatility of ammonia (low pH) and to prevent microbial growth (to prevent breakdown of urea into ammonia and carbon dioxide). This pretreated urine is stored in tanks for weeks to months at a time before a batch is distilled. Distillation occurs in the VCD unit where water and residual VOCs and trace ammonia are separated from the brine (urine, salts, hardness). Water recovery is dependent on a number of factors, with one of the main limitations being salting out of the brine and hardness fouling the unit. The treated urine along with hygiene water and humidity condensate are then passed through a series of air-liquid separators to remove entrained air, multifunction filters to removed suspended and dissolved solids, a catalytic oxidation reactor to remove small chain organic compounds, and ion exchange to remove the rest of the dissolved ions and add iodine to the now potable water. A simple schematic can be found in figure 1 below. 


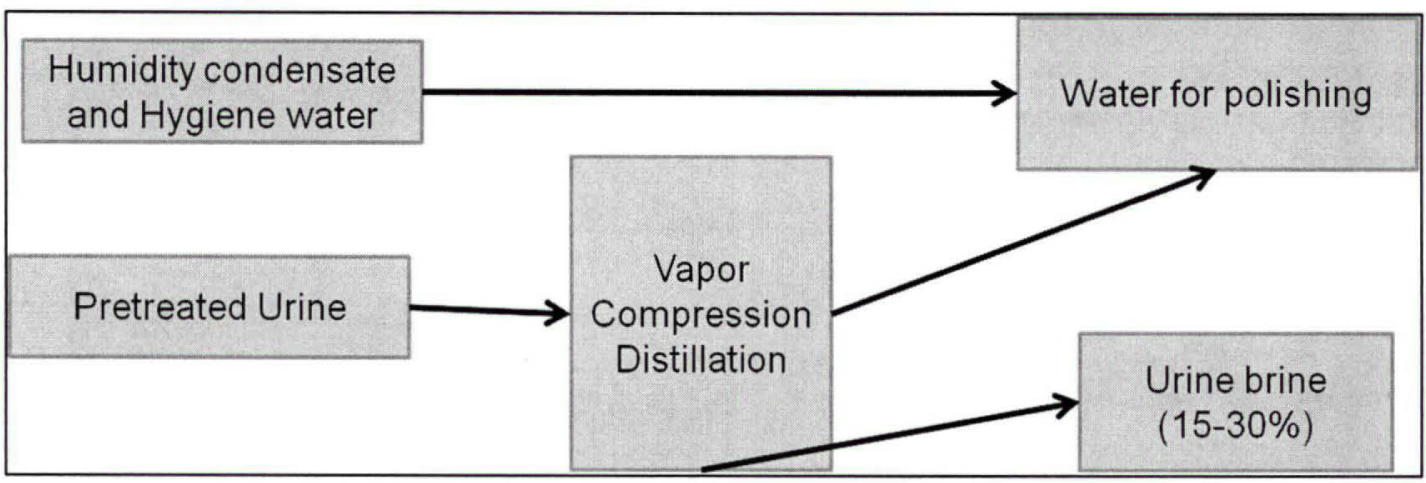

Figure 1: Current basic process flow diagram for ISS waste water treatment and purification

This system allows for many advantages. This architecture uses proven unit operations and delivers high quality water for potable use. This system can handle hygiene water including toothpaste and various soaps unlike previous systems. Urine is purified using vapor compression distillation which is highly energy efficient with low operating temperature (near body temp) and recycles latent heat of vaporization (similar to a regenerative heat exchanger but with phase changes).

However this system has some limitations. Water recovery is poor due to the large amount of liquid brine formed where no more water recovery is possible. The brine itself is highly toxic due to the pretreatment used (chromic acid) and prevents nutrient recovery activities from being performed. There have been times where the pretreatment has failed and microbes were able to multiply and break down the urea to ammonia causing the batch to be lost with zero water recovery. Reliability has been a concern, especially with the vapor compression distillator and its long periods of downtime. Consumable quality is low compared to other systems but still requires pretreatment chemicals, $\mathrm{pH}$ control, filtration beds, catalyst, and ion exchange beds, which are not regenerable in-situ. Implementing hydroponics or brine recovery systems would be impossible with the current pretreatment regime, and this system would require heavy modifications or scrapping to accomplish this. These disadvantages have leaded us to propose a new architecture to handle these challenges.

\section{B. Proposed modular architecture}

In order to handle short term and long term missions with or without hydroponic systems or brine recovery we set out on working on a modular architecture to handle these requirements. To increase water recovery and allow possible nutrient recovery we decided that pretreatment was antithetical to this goal. Instead of stabilizing the urea it made better sense to optimize and regulate its breakdown to ammonia which can then be selectively removed from the wastewater. This allows the ammonia to be used for other purposes (nutrients, refrigeration, pressurizing gas as nitrogen) while simultaneously reducing the dissolved solids in the brine and allowing higher theoretical water recovery before salting out. Combining the wastewater streams allows efficient TOC and TN removal in all the stream and reduces the pressure on the water polisher. Removing the ammonia (and urea) before water recovery should prevent ammonia from reaching the polishing section and reducing the consumable demand. Without urea in the brine, brine recovery unit operations become more feasible and the de-brined brine can be recycled back to water recovery (not shown). The basic scheme can be found in figure 2 below. 


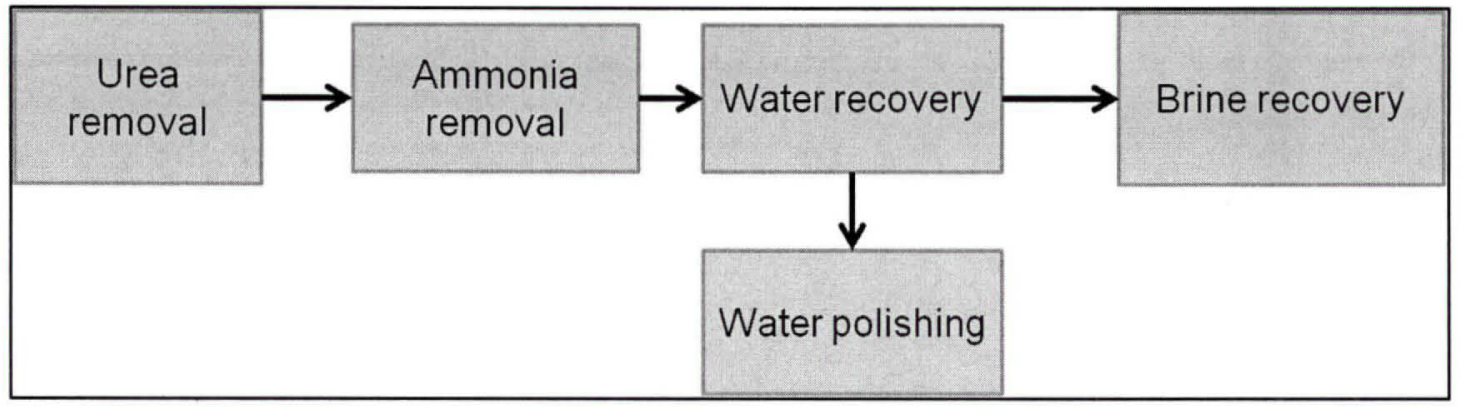

Figure 2: Proposed basic process flow diagram for ISS or extraterrestrial waste water treatment and purification.

The above schema does not require specific unit operations, but allows various unit operations to be auditioned and rotated out to optimize the process. As long as each conversion goes to completion or near completion the system should function. Individual unit operations can be tried for each section and compared to each other to increase conversion, reduce size, lower consumable demand, or increase reliability. To fit with the modularity of the system, the system goals and requirements can change and with unit operation changes can meet those requirements without scrapping the whole system. Some of these changes can be as simple as running a different chemical reaction in a vessel or as complex as adding a plant growth system to augment food supplies. With this flexibility this system will not be immediately obsolete with ever changing mission requirements. By utilizing biological treatment systems that can perform different reactions is the same reactor the "agility" of the system can be increased.

\section{A. Urea hydrolysis bioreactor}

\section{Unit Operation Analysis for waste water pretreatment}

Urea $\left(\mathrm{CO}\left(\mathrm{NH}_{2}\right)_{2}\right)$ is the major component of urine, and contains the large majority of TOC and TN in the wastewater. Urea is nonvolatile and is quite easy to separate using distillation or even membrane separations like reverse osmosis. However, urea has a propensity to break down into its constituent parts due to urease activity from microorganisms. Pretreatment can prevent this by sterilizing the solution but some bacteria can survive the chromic acid and oxone. In addition there are other TOC sources besides urea that are partially made up of VOC's which will end up in the influent to the water polisher. To combat both these problems we look to a bioreactor. Heterotrophic biofilms inside a HFMB enzymatically break down urea and consume TOC to produce ammonia and $\mathrm{CO} 2$ according to the below formula:

\section{$\mathrm{CON}_{2} \mathrm{H}_{4}+\mathrm{H}_{2} \mathrm{O} \rightarrow 2 \mathrm{NH}_{3}+\mathrm{CO}_{2}$ (urease enzyme)}

TOC is severely reduced while the TN balance shifts from primarily urea to almost exclusively ammonia. HFMB are utilized due to their mass transfer and ability to aerate in micro-g environments unlike traditional biological wastewater treatment. These biological reactions occur near ambient temperatures ( $25 \mathrm{C}$ for the ISS) and near Atmospheric pressure. Urea hydrolysis is ussally combined with nitrification/denitrification in a combined bioreactor, but can be performed by itself. The latter is easier to control, more robust, and has a lower residence time compared to the combined approach. After 1248 hours of contact time, the urea is reduced $99 \%$ or more with a pH near 9 with $95 \%$ or greater TOC removal. This high effluent $\mathrm{pH}$ allows downstream ammonia removal which is optimal at alkaline $\mathrm{pH}^{\prime} \mathrm{s}$. Consumable usage is minimal, only oxygen supplied to the Hollow Fibers to aerate the biofilms and increase growth and respiration. No $\mathrm{pH}$ control is needed. A process flow diagram of spacecraft urea hydrolysis HFMB is shown below in figure 3 : 


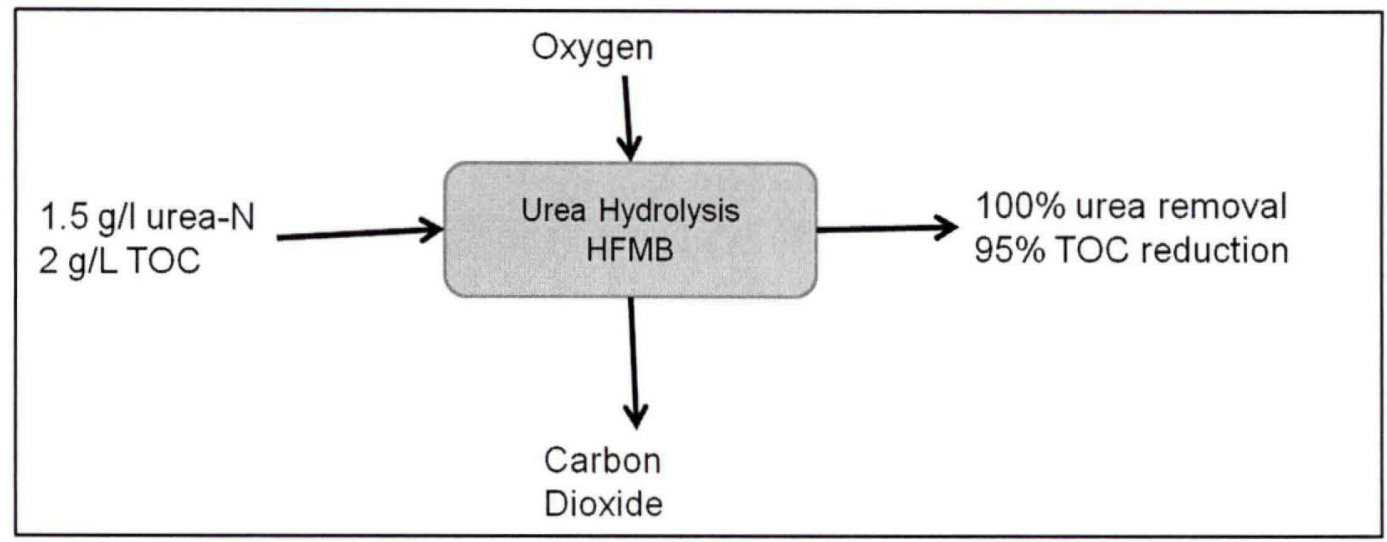

Figure 3: Process flow diagram of Urea hydrolysis HFMB. The waste stream used can be found in (Verostak 2009) under early planetary base waste stream.

Urea hydrolysis can also be performed by traditional physical-chemical methods. To produce the following reactions elevated temperatures at standard pressures are provided without catalysis:

$$
\begin{gathered}
\mathrm{NH}_{2} \mathrm{CONH}_{2}+\mathrm{H}_{2} \mathrm{O} \rightarrow \mathrm{NH}_{2} \mathrm{COONH}_{4} \\
\mathrm{NH}_{2} \mathrm{COONH}_{4} \rightarrow 2 \mathrm{NH}_{3}+\mathrm{CO}_{2}
\end{gathered}
$$

The overall reaction is endothermic, and therefore requires energy to proceed. A CSTR can remove a majority of the urea in under an hour between 100 and $200 \mathrm{C}$ operating temperature. However this process produces water vapor and requires suitable ammonia/water separations which are difficult in micro-g environments. High conversion requires high temperatures, which energy requirement is significantly higher than distillation itself. For now we consider the biological urea hydrolysis process the most feasible for spacecraft utilization.

Urea hydrolysis HFMB holds many advantages compared to pretreatment and physical chemical urea hydrolysis. Controlled Bacterial urea hydrolysis is consistent with $99 \%+$ urea removal with test reactors running for multiple year's straight. Consumable use is low, with no $\mathrm{pH}$ control or other chemical additions (besides oxygen gas delivered to the hollow fibers). Unlike pretreatment or physical-chemical urea hydrolysis, the HFMB can handle VOC's and when a combined wastewater stream is used it can knock down TOC by over $95 \%$; which can significantly reduce the work of downstream polishing. The effluent is high in ammonia and dissolved carbon dioxide which allows nutrient recovery, refrigeration recovery, or atmospheric gas producing activities. Disadvantages of the system include complexity (compared to pretreatment), High $\Theta$ (compared to physical-chemical urea hydrolysis), and slow start up of bioreactor.)

Previous research in urea-hydrolysis bioreactors has focused on combined urea hydrolysis and nitrification/denitrification systems. Current Research has moved towards hollow fiber membrane bioreactors due to their ability to operate in micro-g environments. Recent work has looked into running dedicated urea hydrolysis reactors to optimize this first stage and prevent possible unwanted denitrification and NOx production. Reliability, durability, and Robustness is a priority for future research, with particular interest in having the biological culture survive various qualities (or lack thereof) of influent. 
B.

Borrowing from conventional terrestrial-based waste water treatment, Combined NitrificationDenitrification Bioreactors have been looked at for spacecraft water recovery. Urea hydrolysis can be performed in the same bioreactor or in a previous bioreactor, but only ammonia participates in these reactions. Nitrifying biofilms made up of autotrophic organisms like Nitrosomonas or Nitrobacter then process this ammonia according to the following reactions:

\section{$\mathrm{NH}_{3}+1.5 \mathrm{O}_{2} \rightarrow \mathrm{NO}_{2}^{-}+\mathrm{H}_{2} \mathrm{O}+\mathrm{H}^{+}$(Nitrosomonas or similar autotrophic bacteria) $\mathrm{NO}_{2}^{-}+0.5 \mathrm{O}_{2} \rightarrow \mathrm{NO}_{3}^{-}$(Nitrobacter or similar autotrophic bacteria)}

Note that oxygen is required for these reactions to proceed, so again by using HFMBs oxygen can be provided to these biofilms without mixing/aeration and independent of gravity. Also note that $\mathrm{H}^{+}$is produced in the first reaction which lowers the $\mathrm{pH}$ of the solution, which can knock the reaction out of its most efficient $\mathrm{pH}$ range (7.5-8.5); therefore caustic ( $\mathrm{NaOH}$ or similar) is added to balance and control the $\mathrm{pH}$ to maximize conversion rate. Stoichiometrically $7.14 \mathrm{mg}$ alkalinity is required for every $\mathrm{mg} \mathrm{NH} 3$ removed. Once Nitrate is formed (or even nitrite), it can then be denitrified by the below process:

\section{$\mathrm{NO}_{3}{ }^{-} \rightarrow \mathrm{NO}_{2}{ }^{-} \rightarrow \mathrm{NO}+\mathrm{N}_{2} \mathrm{O} \rightarrow \mathrm{N}_{2}(\mathrm{~g})$ (various bacteria, heterotrophic/autotrophic/anaerobic/aerobic)}

This process traditionally was performed by anaerobic heterotrophic bacteria. However, it has been found that heterotrophic bacteria can denitrify under aerobic conditions as well. Nitrite denitrification is possible using normally autotrophic nitrifying bacteria under certain conditions. A carbon source is required to denitrify in this manner, and requires $1.9 \mathrm{~g}$ methanol per gram nitrogen denitrified It has been shown that urea among the other carbon sources in mixed spacecraft waste water can be used to perform denitrification in a combined stage reactor. Figure 4 indicates four reactor configurations with and without caustic/methanol additions and with and without combined urea hydrolysis. Note that without sufficient alkalinity there will be incomplete ammonia oxidation and without sufficient methanol or other carbon source there will be incomplete denitrification.

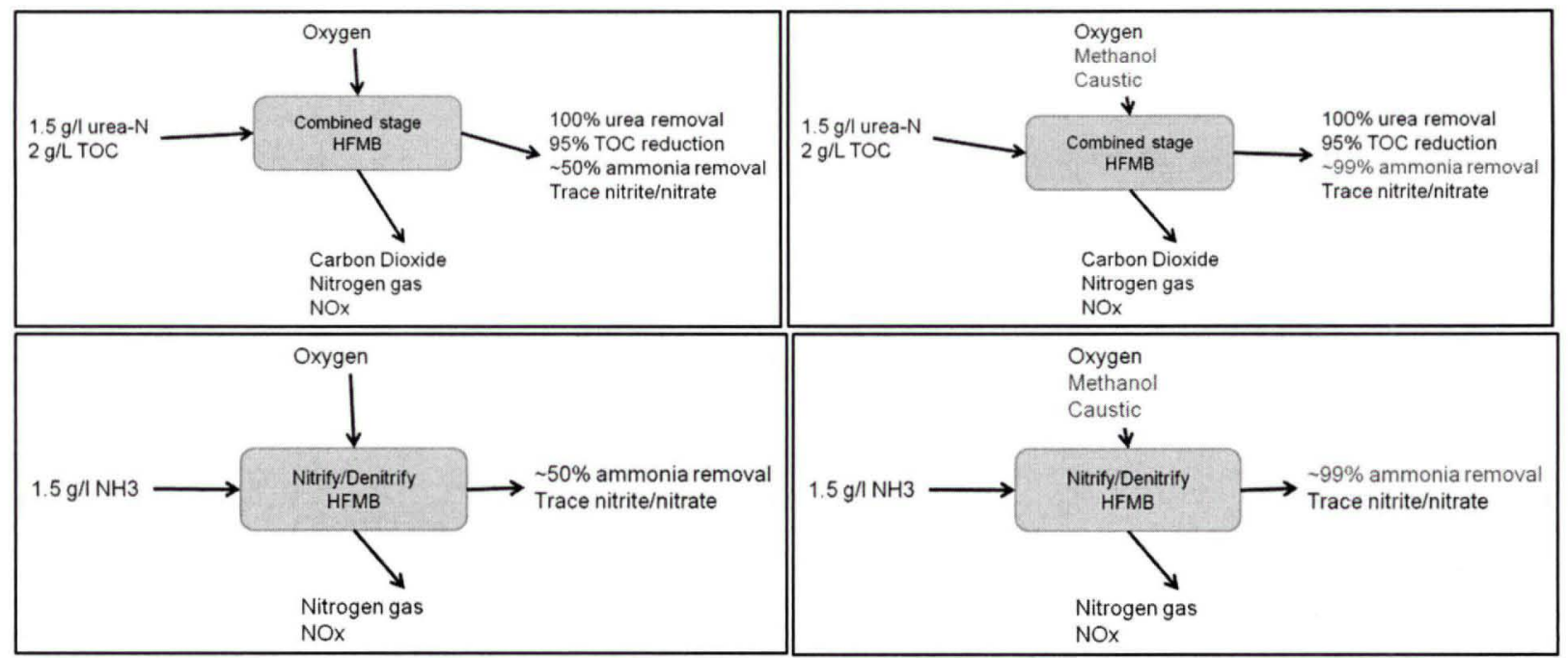

Figure 4: Various configurations of Combined nitrification/denitrification HFMBs. The PFD's on the left refer to alkalinity/carbon source limited systems compared to the right figures which are given 
alkalinity and a carbon source. PFD's on the top combine with the urea hydrolysis stage while the ones on the bottom assume complete urea hydrolysis in a previous HFMB. NOx to N2O)

The main advantage of nitrification-denitrification is simplicity. Using as little as one reactor it is possible to break down urea, then convert ammonia to nitrite/nitrate, then to denitrify to nitrogen gas. There is residual alkalinity and carbon to get significant, but not complete conversion but under this operation no consumables are required. Downsides include low conversion unless consumables are added which adds system complexity and supply mass in the form of alkaline solutions and methanol, which has its own hazards. Incomplete denitrification leads to $\mathrm{N2O}$, which would be a challenge in removing in the gas stream effluent. Our own studies in combined urea hydrolysis/nitrification/denitrification reactors shows long and complex start up with feeding interruptions causing loss of subsequent nitrification. This could be due to the heterotrophic culture feeding off the nitrifying culture during starvation. Start up after starvation has proven to be slow as well. Optimizing operating conditions for vastly different cultures has also proven to be a challenge.

Previous research for spacecraft nitrification/denitrification systems has been coupled with urea hydrolysis above. Terrestrial research has focused on running both reactions in the same reactor by varying aeration and other factors and controlling $\mathrm{N}_{2} \mathrm{O}$ production which on earth is a serious source of greenhouse gas. Further research will be focusing on these terrestrial problems and how they can affect space-based systems. Current research is focused on optimized the current HFMBs for use on future missions.

C.

Nitrate-Nitrification Bioreactor

Nitrogen gas produced by coupled nitrification/denitrification is useful as a pressurizing gas in enclosed environments. However, if nutrient recovery is desired coupled nitrification/denitrification becomes counter-intuitive. We looked for a design that can both stabilize the nitrogenous wastewater while allowing downstream nutrient recovery. Nitrate is a valuable plant nutrient and is non-volatile which should allow it to separate into the brine; unlike ammonia which will end up in the distillate or permeate. Allowing nitrification to go to completion (high nitrate:nitrite selectivity) while preventing denitrification (low influent TOC) would allow these goals to be met. Figure 5 below summarizes the design of this process.

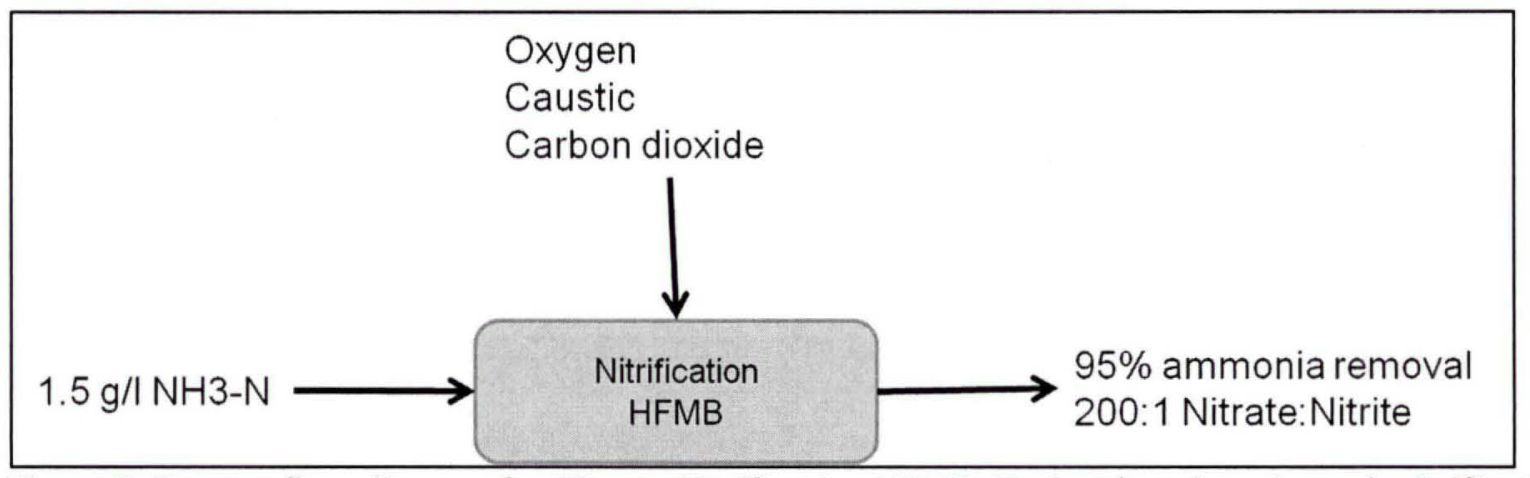

Figure 5: Process flow diagram for Nitrate-Nitrification HFMB. Notice that there is no denitrification in the reactor.

To both maximize ammonia removal and to favor the production of nitrate over nitrate, sufficient oxygen and alkalinity are provided. Optimal $\mathrm{pH}$ for nitrification, especially nitrite oxidation is around 7.5 
and 8.5. Higher $\mathrm{pH}^{\prime}$ s increase the fraction of $\mathrm{NH} 3$ over $\mathrm{NH} 4$ in aqueous solutions and can lead to ammonia toxicity in the biofilm. $\Theta$ is increased to allow nitrification to go to completion (nitrite to nitrate) and to minimize effluent nitrite levels. By utilizing a urea hydrolysis bioreactor upstream of nitrification the urea (and TOC) can be reduced greatly to prevent denitrification. In addition effluent gaseous $\mathrm{CO} 2$ can be plumbed to the downstream nitrification reactor to increase cell mass to the autotrophic nitrifying bacteria. This contrasts to combined stage bioreactors where the urea will be used as the carbon source for denitrification. The end result will be a non-volatile high nitrate wastewater where water and nutrient recovery is facilitated.

The main advantage of the nitrate-nitrification process is that it simplifies the effluent: Instead of a mixture of gaseous and liquid products it concentrates it into nitrate with trace ammonia and nitrite. This can allow simplified downstream operations and allow the gas to be returned to the cabin without any ill effects. Nitrate allows future plant growth, but even without plant growth systems nitrate will be very stable in water recovery operation or storage unless organic compounds are added; contrasting with either urea or ammonia. Disadvantages of the system include complexity and consumables with caustic additions and $\mathrm{pH}$ control compared to other biological ammonia sequestering technologies. Residence time is higher than the combined approach or even Anammox due to time needed to maximize nitrate formation; this would lead to a larger reactor vessel to perform this reaction. Like other biological systems start up time is slow (months) but studies have shown high resiliency to hibernation (lack of feeding). If for some reason the nitrifying culture was lost (due to poisoning or cannibalism by other bacteria/protists) the reactor would need to be re-inoculated and wastewater processing would be delayed months until steady state could be reached. This is a problem for most bioreactors but especially for slow-growing autotrophic bacteria like nitrifiers.

As mentioned above for spacecraft water systems combined stage bioreactors have been preferred for simplicity. Current research at Kennedy Space Center has looked into dedicated nitrate-nitrification HFMBs to try to optimize that reaction independently. Currently they are fed a influent of raw ammonia between 400 and $800 \mathrm{mg}-\mathrm{N} / \mathrm{L}$ along with trace minerals and ran at a 2-day $\Theta$ with $\mathrm{pH}$ control between 7.5 and 8 to satisfactory results. Future studies for Next Generation Life Support will focus on tying together an upstream urea hydrolysis HFMB and optimizing residence time, $\mathrm{pH}$ control, recycle ratio, and other factors. Additional studies are also looking into increasing the robustness of the nitrifying biofilms and increasing start up time or even allowing "hot-starting" by using immobilized fibers.

\section{Anammox Denitrification Bioreactor}

The previous Nitrification methods mentioned are aerobic processes. Until recently it was thought that bio-catalyzed oxidation of ammonia was only possible in aerobic environments. It was later found that there are multiple organisms (bacteria and archea) that can perform ammonia oxidation under anaerobic or anoxic conditions. Under the trademark Anammox licensed to TU Delft, this process is quite different from known aerobic nitrification, using nitrite as a terminal electron acceptor and producing nitrogen gas without an external carbon source:

$\mathrm{NH}_{4}{ }^{+}+\mathrm{NO}_{2}{ }^{-} \rightarrow \mathrm{N}_{2}+2 \mathrm{H}_{2} \mathrm{O}$. (Anammox performing bacteria and archea under anoxic conditions) Anammox performing organisms have extremely slow growth rates, even compared to other autotrophs, and can be as high as a 2 week doubling time. Even with such a slow growth rate Anammox is believed to be responsible for a significant portion of denitrification in aquatic and marine environments. More recently new terrestrial waste water treatment plant designs have looked to incorporate Anammox reactions to save on aeration and consumable costs. Reactor designs used to 
perform Anammox include Sequencing batch reactors and gas-lift bioreactors. Two common reaction schemes include the two stage SHARON/Anammox process and the single stage CANON process.

Single reactor system for high ammonium removal over nitrite (SHARON) is a reaction scheme that nitrifies approximately $50 \%$ of influent ammonia to nitrite to save on consumables and aeration costs. Nitrite levels are controlled by turning on and off aeration and $\mathrm{pH}$ control if there is insufficient alkalinity. The effluent ammonia and nitrite are then sent to an anoxic Anammox reactor where the characteristic reaction takes place and produces nitrogen gas and leaving only trace ammonia/nitrite/nitrate. Mixed spacecraft wastewater without $\mathrm{pH}$ control reaches near $50 \%$ nitrification and under short $\Theta$ gravitates towards nitrite. In short having an HFMB without $\mathrm{pH}$ control taking effluent urea hydrolysis waste water will perform close to a SHARON reactor. Figure 6 below details the process.

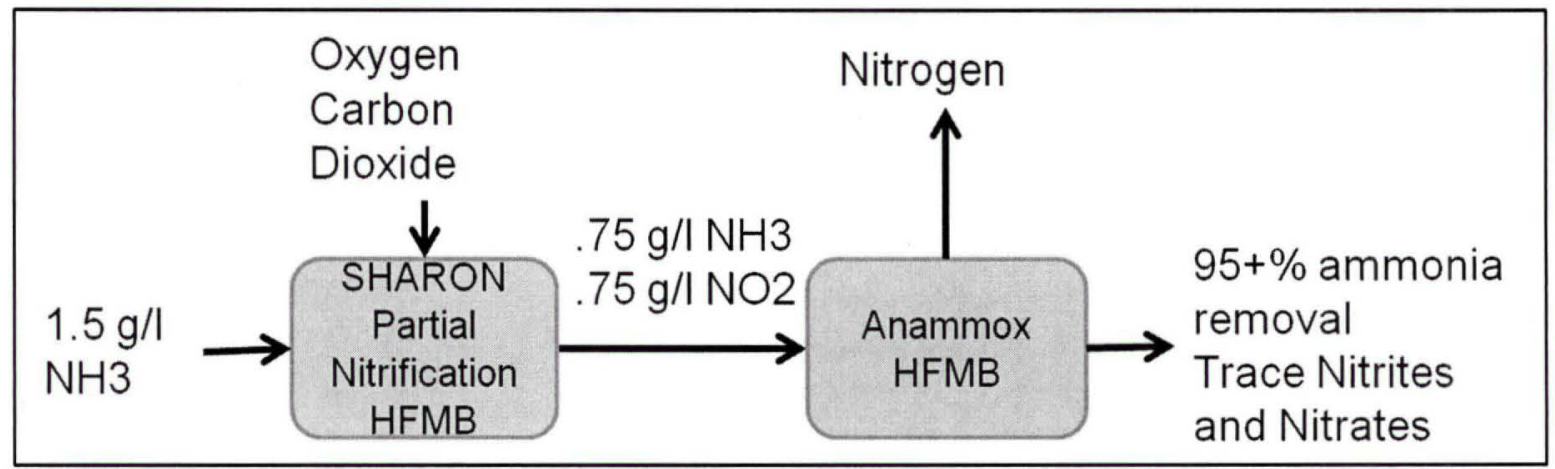

Figure 6: SHARON ${ }^{\circledR}$ Anammox process. Note the lack of $\mathrm{pH}$ control and external carbon source

Completely autotrophic nitrogen removal over nitrite (CANON) process for Anammox is a competing process. Instead of two separate reaction vessels CANON aims to combine the steps in a single reactor. It was found that Anammox organisms are only reversibly inhibited by oxygen and therefore a single stoichiometrically oxygen limited reactor could support both nitrite-nitrification and Anammox without inhibiting the latter. It is thought that the nitrification culture scavenges the limited oxygen to allow the Anammox organisms to thrive. This can materialize as a floc with an outer coating of nitrifiers protecting an inner core of Anammox organisms. On the same token, using aerated hollow fibers in a HFMB, an inner coating of nitrifying biofilm can shield an outer coating of Anammox organisms from oxygen while allowing sufficient mass transfer of ammonia and nitrite as well as other trace minerals. Figure 7 details the basic mass balance of this process. 


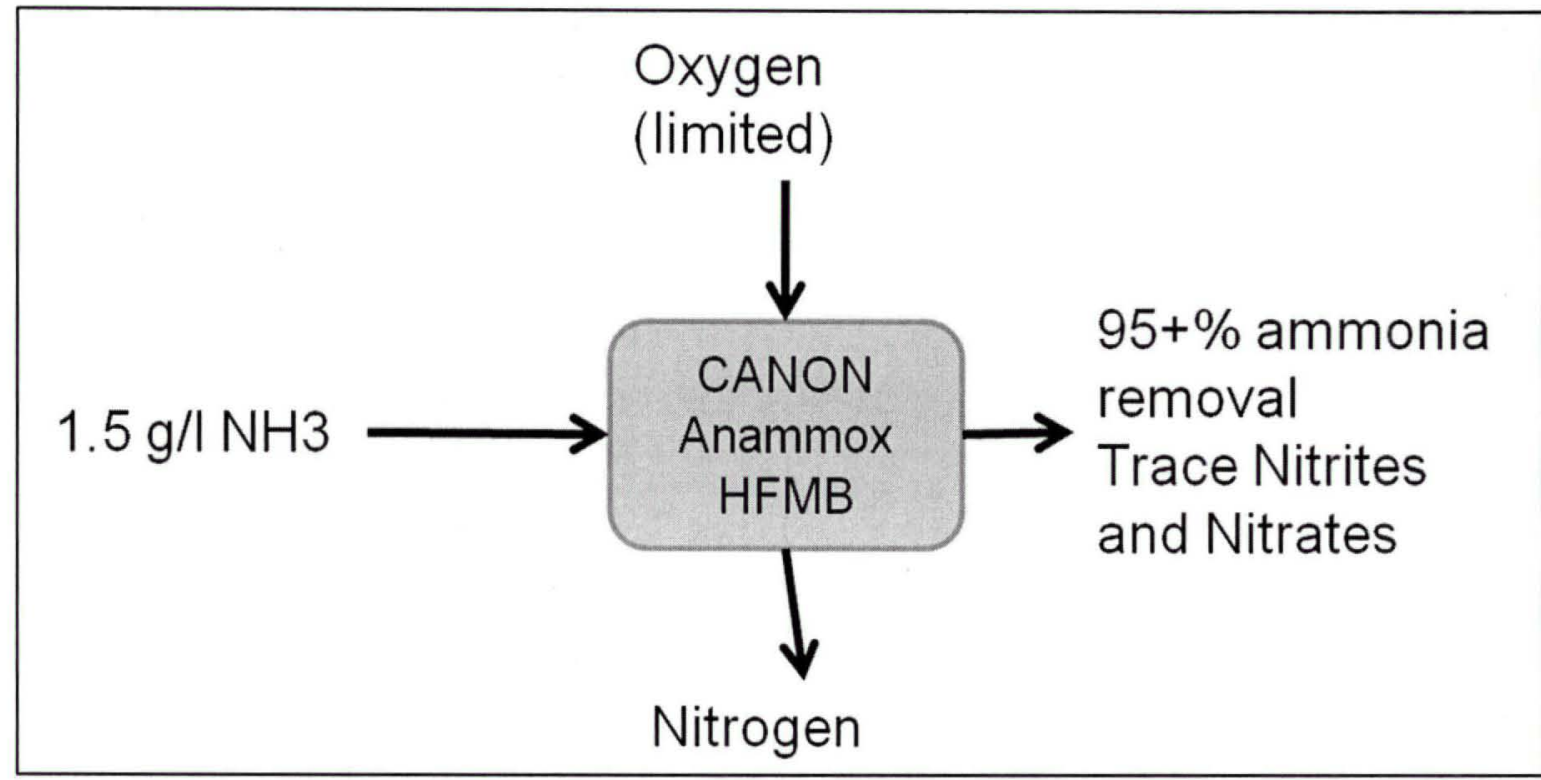

Figure 7: CANON ${ }^{\circledR}$ Anammox process

The main advantage of any Anammox system is the lack of energy and consumables needed to run the reaction. Less oxygen is required as well as no $\mathrm{pH}$ control or external carbon sources needed in most scenarios. Even without consumables conversion is high with over $90 \%$ removal of ammonia reported with high volumetric removal of $\mathrm{N}$ comparable to nitrification/denitrification. The main problem with Anammox systems is the incredibly long start-up time for a reactor or culture. Pilot plants can take years to reach start up while being prone to wash out and die offs. For use on spacecraft the reactor would almost certainly need to be inoculated and mature before being put into orbit. The SHARON configuration suffers from higher complexity (more vessels) than other denitrification systems while CANON is harder to control with two different cultures in the same reactor. Gas handling and two phase flow issues may pop up in zero g applications with the nitrogen gas either diffusing through the hollow fibers or form stationary bubbles in the aqueous portion of the bioreactor.

Anammox is currently in the pilot scale for terrestrial waste water treatment. Due to its low equivalent system mass requirements it shows promise for spacecraft water recovery. However the start up times and fragility of the system have kept it out of discussion for ECLSS architectures. These same difficulties make even lab-scale testing difficult for all but the most determined researcher. Likewise, there have been no reports of attempting to build such a system to handle spacecraft waste water. Key research needs to be performed on biofilm or enzyme immobilization to decrease or eliminate start up time and increase robustness.

\section{E. Regenerative ammonia absorption through struvite reactor}

The processes mentioned so far have been biological in nature. Chemical ammonia removal, specifically absorption; suffers from poor selectivity and poor removal rates and capacities. This has led us to consider a novel ammonia removal technology that harnesses Magnesium Phosphate dibasic trihydrate (MP). When MP exposed to ammonia at elevated $\mathrm{pH}$ in an aqueous solution, it spontaneously reacts to form struvite, a white solid that is primarily known as scale in urea hydrolysis bioreactors:

$\mathrm{MgHPO} 4 * 3 \mathrm{H} 2 \mathrm{O}(\mathrm{s})+\mathrm{NH} 3(\mathrm{I})+3 \mathrm{H} 2 \mathrm{O}(\mathrm{I}) \rightarrow \mathrm{MgNH} 4 \mathrm{PO} 4 * 6 \mathrm{H} 2 \mathrm{O}(\mathrm{s})(\mathrm{pH}$ 8-9, STP)

This reaction can be reversed to yield ammonia and water vapor and regenerate the MP: 
$\mathrm{MgNH} 4 \mathrm{PO}^{*} 6 \mathrm{H} 2 \mathrm{O}(\mathrm{s}) \rightarrow \mathrm{MgHPO} 4 * 3 \mathrm{H} 2 \mathrm{O}(\mathrm{s})+\mathrm{NH} 3(\mathrm{~g})+3 \mathrm{H} 2 \mathrm{O}(\mathrm{g})$ (Elevated temperatures)

The effluent ammonia and water vapor can be used as fertilizer for plant hydroponic growth systems or even purified and injected into ammonia based refrigeration systems commonly used in spacecraft. The resulting MP is then used to absorb more ammonia and can be regenerated repeatedly with minimal substrate loss. MP is extremely selective for ammonia and ignores most other chemical species in waste water. The resulting MAP (along with MP) are sparingly soluble in water and do not increase effluent Magnesium and Phosphate levels. The reactor design is quite open, with fluidized bed reactors (FBRs) popular for similar struvite reaction while plug-flow reactors are untested. Figure 8 below illustrates the Struvite ammonia removal process:

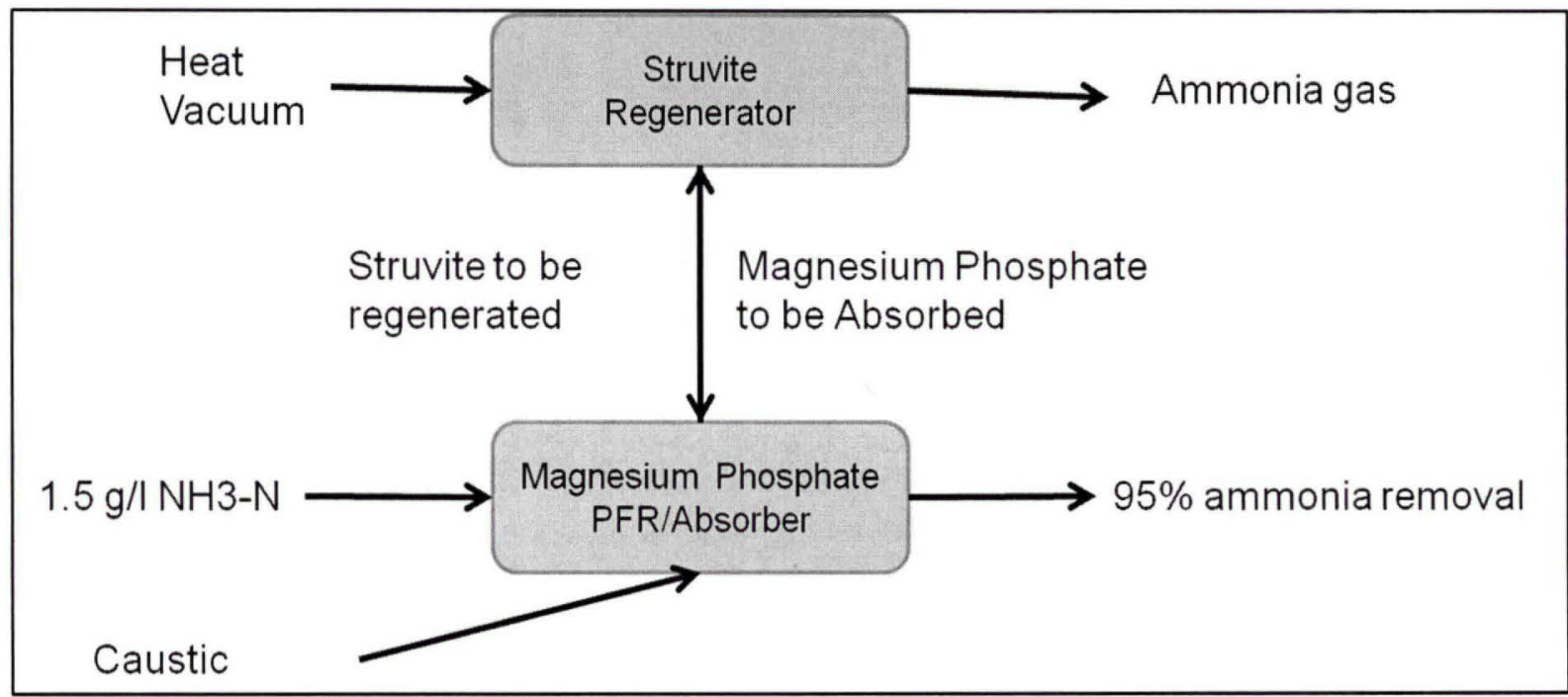

Figure 8: Proposed regenerative ammonia absorber for spacecraft wastewater.

From a mass balance prospective the Struvite absorption process is superficially similar to nitrification/denitrification. Influent aqueous ammonia is supplied along with caustic to keep the $\mathrm{pH}$ between 8 and 9 to increase the reaction rate while the effluent is significantly lower in ammonia and lower in $\mathrm{pH}$. Unlike nitrification no oxygen or HFMB is required; this reaction can take place is a small pipe with filters on each end. Influent and caustic are pumped in until breakthrough of ammonia occurs, the column is regenerated by heat and vacuum to expel the captured ammonia and water vapor, and the fresh column is inserted back into the system.

Advantages compared to biological systems mainly deal with complexity. Struvite absorption requires orders of magnitude less contact times (hours compared to days) and has zero start up time. Performance is instantaneous and spare columns can be used while desorption occurs. Complexity is low since this is simply a packed column of a white powder compared to a bioreactor. Disadvantages include energy and vacuum requirements for regeneration which are higher than comparable bioreactors. Absorption capacity is low (70 mg NH3/g MP) so regeneration is a common occurrence. Ammonia handling post-desorption is also an unresolved issue including possible dehumidifying for refrigeration applications. Organic or bio-fouling could also be a long term issue. Like nitrification struvite formation has a caustic demand that needs to be met to make contact times reasonable and reaction complete.

Research on this specific absorption mechanism has mostly been limited to the beaker scale. Other chemical ammonia removal from wastewater has focused on selective ion exchange resins or even 
Electrochemical Ammonia Reduction through a platinum catalyst (Flynn, Spanish dude. Due to the high selectivity and high regenerability of the struvite process, it remains a candidate for further study. Facets of further study include absorption kinetics, optimized operation, sizing, and deabsorption kinetics and optimization, and feasibility and calculated equivalent system mass, which is only theoretical at this time.

A.

\section{System goals and closing the loop}

One of the major goals of this architecture is modularity. All of the bioreactions mentioned in this paper are capable of being performed in the same modular HFMB. Multiple HFMB can be run in parallel to make up for sizing and $\Theta$ discrepancies. For example for $66 \mathrm{~L} /$ day mixed wastewater (Estimated demand for 6 people on the ISS) a 66L HFMB can be set up to run urea hydrolysis (oxygen, no pH control) while a second stage can perform nitrification to nitrate over 2 days of residence time by using 2-66L HFMB in parallel (oxygen, $\mathrm{pH}$ control). This saves on design costs and makes having spare reactors/capacity easier and more space conserving. In addition, switching a nitrification/denitrification reactor to nitratenitrification can be as simple as reinocculating the system, removing external carbon additions, and adding $\mathrm{pH}$ control. This set up gives unprecedented flexibility and agility for ECLSS. Modular bioreactors are the main driving force in having a flexible architecture that can serve both short term and long term mission scenarios.

\section{B. Hydroponic systems}

Short term missions (LEO, Lunar, and etcetera) have relied on either bringing all their water or recycling a percentage of it. Food has been brought on all missions to date. Plant growth for food crops has been a continued interest to NASA. Along with water recycling, food production from plants can increase endurance of manned missions to allow travel to Mars and further destinations. Plant growth systems for sustainable manned activities in space is still in its infancy, but the food requirements, as well as the water requirements are prohibitively expensive with current launch systems. Current wastewater recovery processes are not designed to work with hydroponic systems, which is evident by the fact that they perform no nutrient recovery and use harsh pretreatment chemicals. One of the goals of this proposed architecture is to only consider processes that can be integrated with hydroponic systems, yet can be used without them. This would allow a system to be launched without a hydroponic system and when sufficient plant growth systems are ready it could be integrated into the water recovery system without major changes. The synergies with these two systems allow higher water recovery and nutrient recycling to allow both systems to flourish.

C.

\section{Brine recovery}

As mentioned above, water recovery has been the priority for wastewater recovery systems. Nutrient recovery has been limited to getting concentrated brine for disposal. Some research has been done on brine dewatering to maximize water recovery. Without food crops to support in an enclosed environment this would be acceptable. However there are more useful chemicals in waste brine than plant fertilizers. For over 100 years the chlor-alkali process has been used on earth to produce useful chemicals from sodium and potassium chloride salts. The basic reaction proceeds as shown:

\section{$2 \mathrm{NaCl}+2 \mathrm{H}_{2} \mathrm{O} \rightarrow \mathrm{Cl}_{2}+\mathrm{H}_{2}+2 \mathrm{NaOH}$ (requires energy) \\ $\mathrm{Cl}_{2}(\mathrm{~g})+\mathrm{H}_{2}(\mathrm{~g}) \rightarrow 2 \mathrm{HCl}(\mathrm{g})$ (exothermic in an aqueous solution)}

This process converts the previous "waste product" of sodium (or potassium) chloride into sodium hydroxide (caustic) and hydrochloric acid. This is an electrolysis process using membranes similar to those used in fuel cells (nafion). These chemicals can be used for upstream wastewater pretreatment (nitrification and struvite require caustic) while $\mathrm{HCL}$ can be used to stabilize waste water in case of 
process failure. Ion exchange resins used for polishing and fertilizer removal from wastewater require $\mathrm{HCL}$ and $\mathrm{NaOH}$ to be regenerated as well. This process basically converts the mass consumable demand into an energy cost (which is supplied by solar panels or similar renewable source).

The brine needs pretreatment before chlor-alkali can be performed. Spacecraft mixed wastewater brines contain magnesium, calcium, phosphate, sulfates, and possibly nitrates (if nitrification to nitrate is used). Selective ion exchange resins that can be regenerated by either $\mathrm{HCL}$ or $\mathrm{NaOH}$ can allow the sequestering of these chemical species to allow chloralkali to function. The produced acids and bases allow this sequestering to be a regenerable process while the regenerate from the resins (KNO3, $\mathrm{K} 2 \mathrm{HPO} 4, \mathrm{~K} 2 \mathrm{SO} 4, \mathrm{MgCl} 2, \mathrm{CaCl}$ ) are valuable plant nutrients. The effluent brine from these processes (ion exchange and chloralkali) can then be recycled to the water recovery system for higher performance.

D. Near term, short mission, open loop operation

Using this system architecture for a near term short duration mission (LEO) we can shift the process to produce potable water and a pressurizing gas (nitrogen for the living compartment or ammonia for the refrigeration systems) For short term missions plant systems would not be needed so nutrient recovery would be optional. Acids and bases can be produced to lower the consumable demand on the upstream processes while increasing water recovery marginally. Nitrification/denitrification or Anammox can be considered for taking nitrogenous waste products and converting them to pressurizing gas while struvite can be considered for ammonia for refrigeration. As mentioned above the same HFMBs can be used for either biological reaction. Figure 9 indicates the system architecture with possible technologies that can meet these goals.

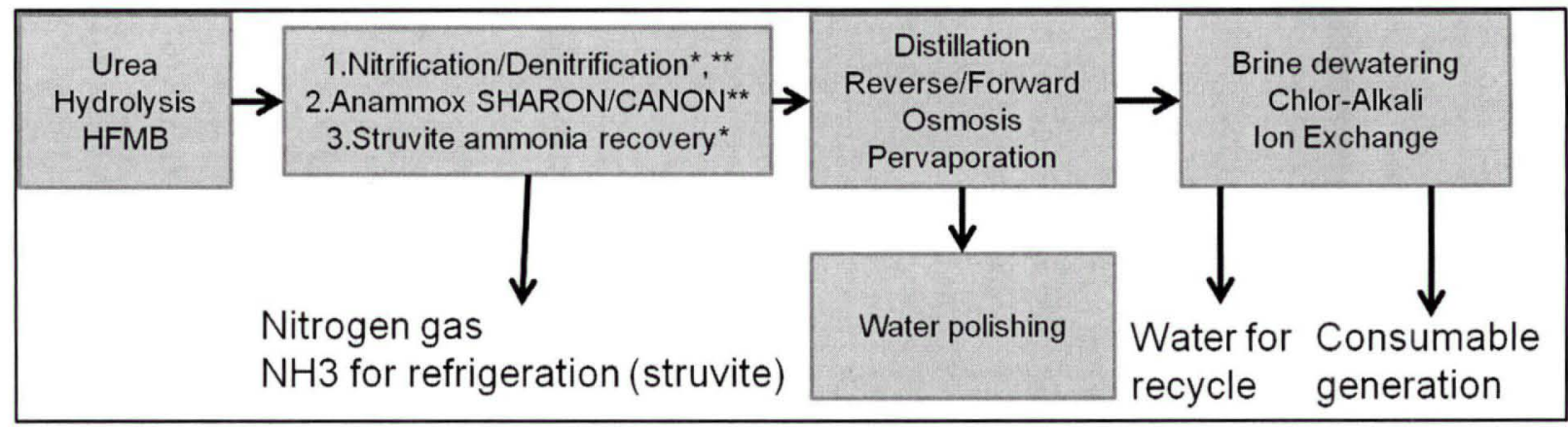

Figure 9: Possible technologies integrated for a near-term water recycler. ${ }^{*}=$ Caustic required while ${ }^{* *}=$ oxygen required for operation.

\section{E. Long term, long mission, closed loop operation}

For Long term mission considerations, food production becomes more important from a launch cost point of view. Taking the same basic infrastructure above, we can convert from gas production to nutrient recovery. Instead of nitrogen gas we can produce soluble nitrate salts that can be recovered for plant fertilizer. Instead of adding ammonia to the refrigeration system we can divert it to a hydroponic system. Nutrients (besides sodium which is recycled internally in the system) are diverted to plant growth and recovered using plant leaching technologies and ran through the same waste water process. Consumables (for water treatment and plant growth) are minimized by this total recovery system. Figure 10 indicates the system architecture with possible technologies that can meet these goals. 


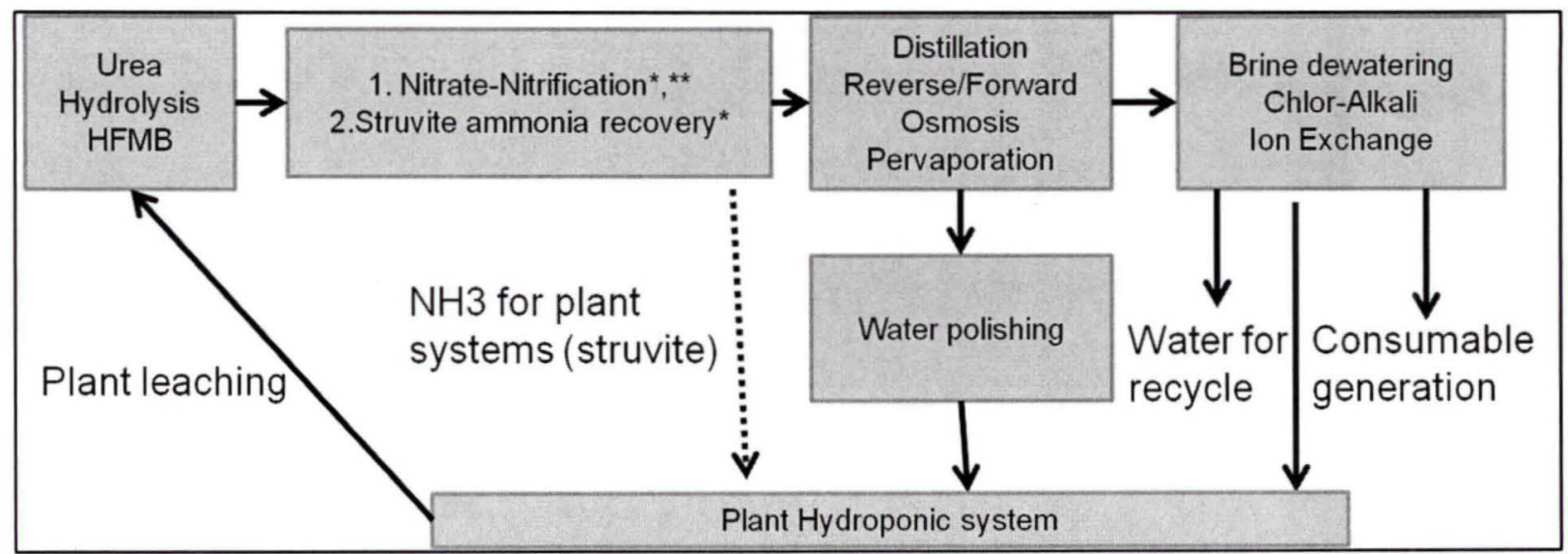

Figure 10: Possible technologies integrated for a far-term water recycler with plant growth systems *=Caustic required while ${ }^{* *}=$ oxygen required for operation.

\section{v. Conclusion}

This paper has presented an overview on nitrogenous waste water removal options (some established and some novel) while proposing new system architectures to handle them. With these options, it should be possible to construct a system that can meet the mission's needs, either for short term excursions to LEO like the ISS or longer term multi-year missions to Mars. Plant growth systems are still at low TRL currently but future waste water recovery systems should be able to handle if not become synergistic with them. Current waste water recovery systems are not up to this task so new systems using new unit operations should be considered. Further research is proceeding for established and novel unit operations to gather feasibility and optimal conditions to make this a reality. 